\section{Stéphane Viville Chantal Rabourdin- Combe}

\title{
La chaîne invariante: son rôle et sa fonction dans la réponse immunitaire spécifique
}

La chaîne invariante, protéine monomorphe étroitement associée aux molécules du complexe majeur d'histocompatibilité (CMH) de classe II, existe sous différentes formes, toutes synthétisées à partir d'un même gène. Dix ans après sa découverte, son intervention dans la réponse immunitaire spécifique est clairement établie. Les expériences in vitro avaient permis de démontrer son rôle dans l'assemblage des chaînes $\alpha$ et $\beta$ des molécules du CMH de classe II, dans leur transport du réticulum endoplasmique vers les endosomes et dans la présentation de la majorité des antigènes par les molécules du CMH de classe II. Récemment, la création de souris déficientes en chaîne invariante a permis de confirmer certains résultats obtenus in vitro et d'étudier son rôle in vivo.

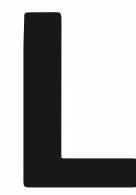

es lymphocytes $\mathrm{T}$, via leur récepteur (TcR), reconnaissent un complexe bimoléculaire formé d'un peptide associé à une molécule codée par le complexe majeur d'histocompatibilité (CMH) de classe I ou de classe II. Les molécules du $\mathrm{CMH}$ de classe II (CMH-II) sont des hétérodimères constitués de deux chaînes protéiques polymorphes $\alpha$ et $\beta$ exprimées à la surface des lymphocytes B, des macrophages, des cellules de Langerhans, des cellules épithéliales thymiques et des lymphocytes $\mathrm{T}$ humains activés. Au cours de leur biosynthèse, les molécules du CMH-II sont transitoirement associées à une troisième protéine monomorphe appelée chaîne invariante (Ii) ou chaîne $\gamma$ ou encore CD74. La chaîne Ii a été iso- lée pour la première fois par Jones et al. chez la souris grâce à des techniques d'immunoprécipitation utilisant des anticorps spécifiques des molécules du CMH-II de souris et un marquage métabolique des protéines cellulaires. Quel que fût l'haplotype des molécules du CMH-II, une glycoprotéine monomorphe, et donc nommée invariante, de masse moléculaire $31 \mathrm{kDa}$, était systématiquement coprécipitée avec les chaînes $\alpha$ et $\beta$ constitutives des molécules du CMH-II. [1]. A la même époque, une protéine très similaire était décrite chez l'homme [2].

\section{Invariante,}

la chaîne invariante?

La chaîne li est une protéine transmembranaire de type II, conservée 


\section{RÉFÉRENCES}

1. Jones PP, Murphy DB, Hewgill D, McDevitt HO. Detection of a common polypeptide chain in I-A and I-E sub-region immunoprecipitates. Immunol Chem 1979; 16 : $51-60$.

2. Charron D, McDevitt HO. Analysis of HIA-D region-associated molecules with monoclonal antibody. Proc Nall Acad Sci USA $1979 ; 76: 6567-71$.

3. Strubin M, Berte C, Mach B. Alternative splicing and alternative initiation of translation explain the four forms of the la antigen-associated invariant chain. $E M B O$ / $1986 ; 5: 3483-8$

4. Koch N, Lauer W, Habicht J, Dobberstein B. Primary structure of the gene for the murine Ia antigen-associated invariant chains (Ii). An alternatively spliced exon encodes a cysteine-rich domain highly homologous to a repetitive sequence of thyroglobulin. $E M B O$ J $1987 ; 6: 1677-83$.

5. Quill H, Schwartz BD. Invariant proteins associated with Guinea-pig Ia antigens. Mol Immunol $1983 ; 20$ : 1333-45.

6. Mehringer JH, Harris MR, Kindle CS, McCourt DW, Cullen SE. Characterization of fragments of the murine Ia-associated invariant chain. J Immunol 1991; 146 920-7.

7. Teyton L, Peterson PA. Invariant chain, a regulator of antigen presentation. T Cell Biol 1992 ; 2 : 52-6.

8. Naujokas, MF, Morin M, Anderson MS, Peterson M, Miller J. The chondroitin sulfate form of invariant chain can enhance stimulation of $\mathrm{T}$ cell responses through interaction with CD44. Cell 1993; 74 : $257-68$.

9. Cleasson-W'elsh L, Barker PE, Larhammar $\mathrm{D}$, el al. The gene encoding the human class II antigen-associated $\gamma$ chain is located on chromosome 5. Immunogenetics 1984 ; 20 : 89-93.

10. Richards JE, Pravtcheva DD, Day C. Ruddle FH, Jones PP. Murine invariant chain gene : chromosomal assignment and segregation in recombinant inbred strains. Immunogenetics 1985; 22: 193-9.

11. Collins T, Korman AJ, Wake C.T, et al. Immune interferon activates multiple class II major histocompatibility complex genes and associated invariant chain gene in blasts. Proc Nall Acad Sci USA 1984; 81 : 4917-21.

12. Rahmsdorf $\mathrm{HJ}$, Koch $\mathrm{N}$, Mallick $\mathrm{U}$ Herrlich P. Regulation of MHC class II invariant chain expression: induction of synthesis in human and murine plasmocytoma cells by arresting replication. $E M B O$ J $1983 ; 2: 811-6$.

13. Acolla, RS, Carra (;, Buchegger F, Carrel S, Mach JP. The human la-associated invariant chain is synthesized in Ia negative $B$ cell variants and is not expressed on the cell surface of both Ia negative and Ia positive parental cells. I Immunol 1985 134: 3265-71.

14. Lombart-Platet S, Bertolino P, Gimenez C, el al. Invariant chain expression similarly controls presentation of endogenously synthesized and exogenous antigens by MHC; class II molecules. Cell Immunol 1993 148: 60-70

15. Kvist S, Wiman K, Claesson L, Peter son PA, Dobberstein B. Membrane insertion and oligomeric assembly of HLA-DR histocompatibility antigens. Cell 1982; 29 : $61-9$

16. Lamb CA, Cresswell P. Assembly and transport properties of invariant chain tri mers and HLA-DR-invariant chain complexes. I Immunol 1992; 148 : 3478-82.

17. Roche PA, Marks MS, Cresswell P. Formation of a nine-subunit complex by $\mathrm{HI}$ class II glycoproteins and the invariant chain. Nature 1991 ; 354 : 392-4.

18. Marks MS, Cresswell P. Invariant chain associates with HI A class II antigens via its extracytoplasmic region. / Immunol 1986 136: 2519-25.

19. Rudensky A, Preston-Hurlburt P, Hong SC, Barlow A, Janeway CA. Sequence analysis of peptides bound to MHC class II molecules. Nature 1991; 353: 622-7.

20. Hunt D, Michel H, Dickinson TA, et al. Peptides presented to the immune system by murine class II major histocompatibility complex molecule I-Ad. Science 1992 ; 256 : 1817-20.

21. Riberdy JM, Newcomb JR, Surman MJ Barbosa JA, Cresswell P. HLA-DR molecules from an antigen-processing mutant cell line are associated with invariant chain peptides. Nature 1992; 360 : 474-7. au cours de l'évolution (la similitude est de l'ordre de $70 \%$ à $80 \%$ entre les protéines humaines et murines), qui est exprimée sous de multiples formes $[3,4]$. Chez la souris, il existe deux formes majeures nommées d'après leur masse moléculaire p3l et p4l; chez l'homme, deux formes supplémentaires, p33 et p43, sont également synthétisées (figure 1). Le clonage de l'ADNc et du gène codant pour li a permis de mieux comprendre l'origine de toutes ces protéines apparentées.

Les formes p31 et p4l résultent d'un épissage alternatif de l'ARN prémessager de Ii et diffèrent par leur extrémité C-terminale; seule la forme p4l contient l'exon (6b), exon particulièrement riche en proline, qui code pour 64 acides aminés et présente une analogie de séquence significative $(40 \%)$ avec les éléments répétés de la thyroglobuline [4]. La forme p31 est plus abondante que la forme p41, selon un rapport qui varie de 5 à 10 . Chez l'homme, les formes p33 et p43 sont dues à l'existence d'un deuxième site d'initiation de la traduction, en phase de lecture et en amont du premier, responsable de la synthèse de 16 acides aminés supplémentaires à l'extrémité Nterminale. Ces deux formes sont moins exprimées que les formes p31 et p4l et la forme p43, qui est la forme la plus minoritaire, est rarement mentionnée dans la littérature. D'autres formes secondaires, p29, p25 et p12 provenant de la protéolyse des précédentes, apparaissent au cours du transport de Ii dans la cellule [5]. La forme p25 serait produite au niveau du réticulum endoplasmique [6] et la précision de coupure suggère la participation d'une protéase spécifique [6]. La forme p29 serait l'un des premiers produits de la dégradation de Ii au niveau des vésicules endosomiques et la forme pl2 l'un des produits finaux [6].

En outre, Ii subit diverses modifications post-traductionnelles au cours de son transport vers les endosomes [7]. Les premières concernent des glycosylations sur deux asparagines (N-glycosylation) pour les formes courtes et sur quatre pour les formes les plus longues. Elles concernent aussi deux O-glycosylations 
(au niveau de sérine ou de thréonine) qui, tout comme les $\mathrm{N}$ glycosylations, subissent l'adjonction d'acide sialique lors du transport de Ii à travers l'appareil de Golgi. D'autres modifications posttraductionnelles prennent place comme: (1) l'adjonction, pour un pourcentage limité des molécules de Ii (2\% à $5 \%$ ), à la partie C-terminale de la protéine d'un glycosaminoglycane de type chondroïtine sulfate. Ces molécules de Ii sont exprimées à la surface cellulaire en association avec les chaînes $\alpha$ et $\beta$ des molécules du CMH-II ; (2) la phosphorylation au niveau de résidus sérine cytoplasmiques; (3) la palmitoylation d'une cystéine cytoplasmique.

Les rôles des différentes formes protéiques et des modifications posttraductionnelles restent à définir. Cependant, il a été récemment démontré que la faible proportion de Ii exprimée à la surface sous la forme chondroïtine sulfate jouerait le rôle de molécules accessoires puisque l'interaction de ces formes avec les molécules CD44 faciliterait l'interaction entre la cellule présentatrice de l'antigène et la cellule T [8].

\section{Expression du gène li}

Bien que le gène codant pour Ii soit situé hors du locus du CMH, sur le chromosome 5 chez l'homme [9] et sur le chromosome $18 \mathrm{chez}$ la souris [10], son expression tissulaire est identique à celle des gènes codant pour les molécules du CMH-II. Tout comme les gènes des molécules du CMH-II, le gène codant pour Ii est inductible par l'interféron $\gamma$ et par l'interleukine 4 [11]. Toutefois, au moins chez la souris, alors que le

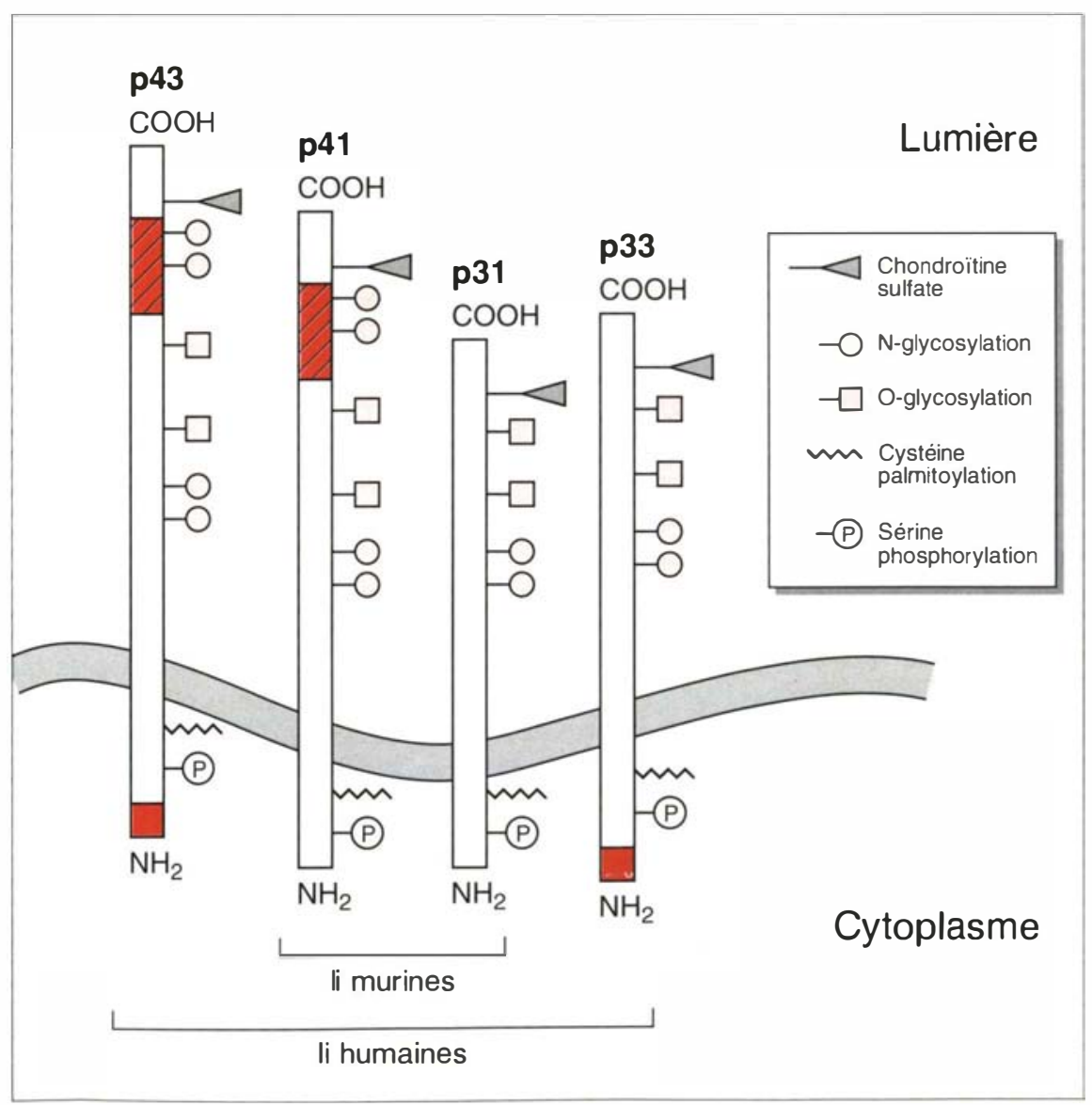

Figure 1. Schéma représentant les différentes formes codées par le gène li chez l'homme et chez la souris. Chez l'homme et chez la souris, par suite d'un épissage différentiel au niveau de l'exon $6 b$, le gène li code pour deux protéines: les formes p31 et p41. Chez l'homme, les formes p33 et p43 sont également produites par utilisation d'un second site d'initiation de la traduction.

$\mathrm{m} / \mathrm{s} n^{\circ} 2$ vol. 10, férier 94 gène Ii s'exprime au niveau des lymphocytes B, il ne s'exprime pas au niveau des lymphocytes pré-B et des plasmocytes [12]. A l'inverse, certaines cellules transformées perdent l'expression des molécules du CMH-II sans perdre l'expression de Ii [13] et les cellules L de souris, lignée de fibroblastes, expriment, dans certaines conditions de culture, la chaîne Ii et non les molécules CMH-II [14].

\section{Biosynthèse de la chaîne li}

L'assemblage des chaînes $\alpha$ et $\beta$ des molécules du CMH-II et de Ii se fait rapidement après leur synthèse au niveau du réticulum endoplasmique [15] où Ii se trouve en large excès par rapport aux chaînes $\alpha$ et $\beta$ et peut donc s'y trouver sous forme libre [15]. Un trimère formé de trois chaînes Ii associées par leur extrémité C-terminale se formerait d'abord, puis les chaînes $\alpha$ et $\beta$ des molécules du CMH-II, déjà sous forme de dimères, prendraient place [16], formant finalement, comme le suggèrent Roche et al., un nonamère constitué de trois molécules de Ii, de trois chaînes $\alpha$ et de trois chaînes $\beta$ [17] (figure 2). Les différentes formes de Ii peuvent participer à la formation de ce complexe, ainsi que les différentes formes alléliques des molécules du CMH-II humaines [17]. L'interaction entre chaînes Ii libres et/ou complexées aux molécules du CMH-II met en jeu les extrémités C-terminales des molécules Ii [18] et des résultats récents suggèrent que la région 81-109 de Ii (exon 3) serait également impliquée dans l'interaction avec les molécules du CMH-II (Bertolino et al., soumis). Il est intéressant de noter que parmi les peptides élués des molécules du CMH-II purifiées sont présents des peptides de Ii, de longueur variable, recouvrant la région 80-120 et ce, dans le cas des molécules ${\mathrm{I}-\mathrm{A}^{\mathrm{b}}}^{\mathrm{b}}[19], \mathrm{I}^{\mathrm{A}} \mathrm{A}^{\mathrm{d}}[20]$, HLA-DR3 [21] et HLA-DR1 [22]. Puisque ces peptides correspondent à une région de Ii proche de la membrane, il est peu probable qu'ils interagissent avec la niche à peptides située à l'extrémité C-terminale des molécules du CMH-II [23], mais 


\section{RÉFÉRENCES}

22. Chicz RM, Urban RC; I ane WS, et al Predominant naturally processed peptides bound to HIA-DRl are derived from MHC:-related molecules and are heteroge neous in size. Nature 1992; 358: 764-8.

23. Hunt DF, Henderson RA, Shabanowitz J, et al. (haracterization of peptides bound to the class I MHC molecule HLA-A2.1 by mass spectrometry. Srience 1992; $255^{\circ}$ 1261-3.

24. Roche PA, Cresswell P. Invariant chain association with HLA-DR molecules inhibits immunogenic peptide binding. Nature 199()$; 345: 615-8$.

25. Roche PA, Teletski C., Karp DR, et al Stable surface expression of the invariant chain prevents peptide presentation by HIA-DR. EMBO J 1992; 11 : 2841-7.

26. Lavet (:, Germain RN. Invariant chain promotes egress of poorly expressed haplotype-mismatched class II major histocompatibility complex $A \alpha \mathrm{A} \beta$ dimers from the endoplasmic reticulum/ cis-Colgi compartment. Proc Natl Acad Sici USA 1991; 88 2346-.5().

27. Viville S, Neefjes J, Lotteau V, et al. Mice lacking the MHC class II-associated invariant chain. Cell 1993; 72 : 635-48.

28. Guagliardi LE, Koppelman B, Blum JS, et al. Co-localization of molecules involved in antigen processing and presentation in an early endocytic compartment. Nature 199()$; 343: 133-9$.

29. Peters PJ, Neefjes IJ, Oorschot $V$, Ploegh HL, (jeuze HJ. Segregation of MHC. class II molecules from MHC class 1 molecules in the Colgi complex for transport to lysosomal compartment. Nature 1991 ; 349: 669-76.

30. Lotteau V, Teyton I, Peleraux A, el al. Intracellular transport of class II MHC. molecules directed by invariant chain. Nature 199(); 348: 6()()-5.

31. Bakke O, Dobberstein B. MHC class IIassociated invariant chain contains a sorting signal for endosomal compartment. Cell 199(); 63: 7()7-16.

32. Simonis S, Miller I, Cullen SE. The role of Ia-invariant chain complex in the postranslational processing and transport of Ia and invariant chain glycoproteins. I ce domaine pourrait correspondre au site d'interaction de Ii avec les chaînes $\alpha$ et $\beta$ des molécules du CMH-II. Toutefois, l'interaction entre Ii et molécules du CMH-II dans le réticulum endoplasmique a pour conséquence une inhibition de la fixation de peptides par les molécules du CMH-II, assurant ainsi une dichotomie dans la présentation des antigènes entre les CMH-I et les molécules du CMH-II [7]. Ainsi Roche et al., à partir de complexes molécules du CMH-II/Ii purifiés, ont montré que Ii inhibe la fixation d'un peptide dérivant de l'hémagglutinine de la grippe sur les molécules HIADR5 [24]. De même, l'expression à la surface cellulaire de complexes CMH-II/Ii tronquée inhibe la présentation antigénique de différents peptides par les molécules HIA-DR5 [25]. Les résultats expérimentaux disponibles à ce jour ne permettent pas de comprendre le mécanisme moléculaire de cette inhibition: s'agit-il d'une inhibition compétitive due à la présence d'une partie de Ii dans la niche ou d'une inhibition indirecte due à une modification de la niche à peptide à la suite de la fixation de Ii hors de la niche?

En outre, un rôle de Ii dans l'assemblage et/ou le repliement des molécules du CMH-II dans le réticulum endoplasmique a été suggéré [26], car certains haplotypes de molécules du CMH-II ne s'associent pas en absence de Ii. Cela a été confirmé par les travaux de Viville et al. sur les souris déficientes en Ii (souris $\mathrm{I}^{\circ}$ ), obtenues par recombinaison homologue $\left(\mathrm{m} / \mathrm{s} n^{\circ} 3\right.$, vol. 9, $p$. 350), qui ont montré que les molécules du CMH-II d'haplotype $\mathrm{H}-2^{\mathrm{b}}$ ne s'assemblent pas en absence de Ii, alors que les molécules du CMH-II d'haplotype $\mathrm{H}-2^{k}$ s'apparient aussi bien en absence qu'en présence de Ii [27]. La formation du nonamère Iimolécules du CMH-II permet le transport de celui-ci du réticulum endoplasmique vers les endosomes [28] et/ou les lysosomes [29]. La délétion progressive des premiers résidus des formes p31 et p33 a permis de cartographier un signal de rétention dans le réticulum endoplasmique dans les quinze premiers résidus de la p33 et un signal de ciblage et/ou de rétention dans les endosomes entre les résidus 12 et 15 de la forme p31 [30, 31]. La présence d'un motif, dans les onze premiers résidus de la forme p31, est responsable de la rétention de la p31 dans le réticulum endoplasmique. En effet, des molécules de Ii tronquées (c'est-à-dire ne possédant pas la région 1-11) sont mieux exprimées à la surface. La sortie de la p31 du réticulum endoplasmique est facilitée par son interaction avec les molécules du CMH-II ; dans ce cas, il est probable que l'interaction de la p31 avec les molécules $\mathrm{CMH}$ II masque le motif de rétention [32].

Les premières preuves du rôle de Ii dans le transport des molécules du CMH-II viennent de l'étude de la glycosylation des molécules du CMH-II en présence ou en absence de Ii. La glycosylation est incomplète et le transport des molécules du CMH-II plus lent dans des cellules qui ne synthétisent pas Ii $[33,34]$. L'association, dans le réticulum endoplasmique, des protéines de stress (GRP94 et ERp72) aux molécules du CMH-II synthétisées en absence de Ii suggère un rôle de protéine chaperon pour Ii puisqu'elle évite une agrégation des molécules du CMH-II dans le réticulum endoplasmique [35]. Cependant, le rôle de Ii dans le transport des molécules du CMH-II a longtemps été ignoré car des cellules transfectées avec des gènes des CMH-II et ne synthétisant pas de Ii expriment quand même des molécules du CMH-II à leur surface [36]. Toutefois, ces études font appel à un tri cellulaire basé sur l'expression des molécules du CMH-II à la surface et conduisant donc inévitablement à une sélection de transfectants stables exprimant des molécules du CMH-II. L'étude de souris déficientes en Ii a clairement démontré le rôle de Ii dans l'expression des molécules du CMH-II à la surface cellulaire. En effet, l'analyse par cytométrie de flux montre que l'expression à la surface cellulaire des molécules du CMH-II chez ces souris $\mathrm{Ii}^{\circ}$ est très réduite, et certainement due à un blocage dans le réticulum endoplasmique puisque la majorité des molécules du CMH-II ne possèdent pas de sucre com- 


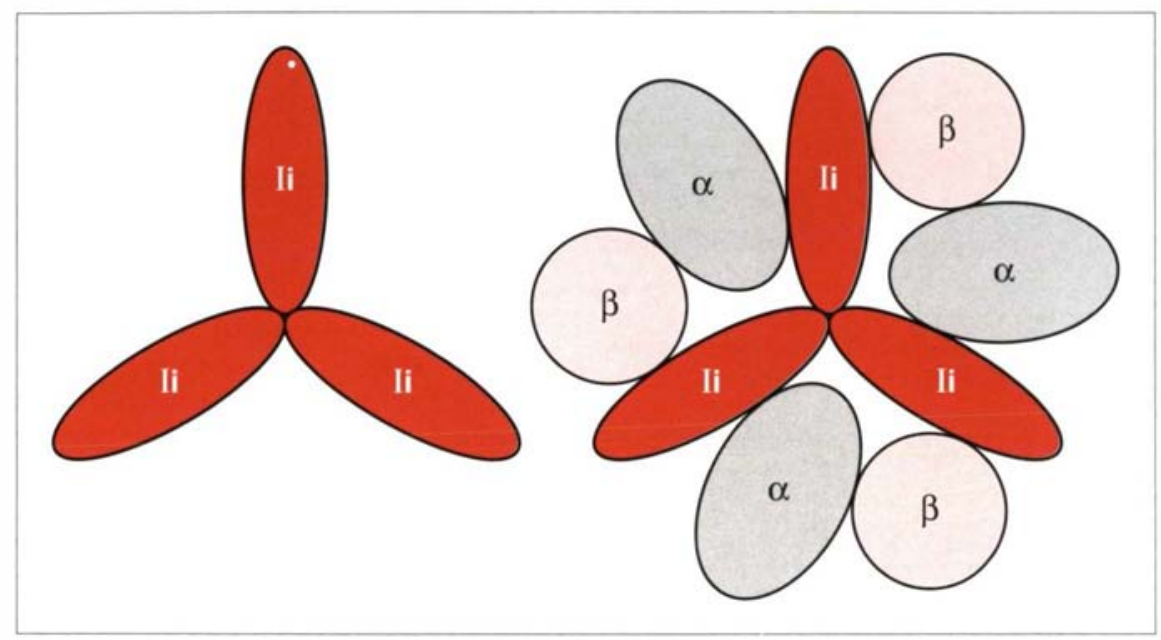

Figure 2. Schéma de la structure hypothétique du trimère de li et du nonamère formé de 3 chaînes $\alpha$, de 3 chaînes $\beta$ et de 3 chaînes li. (D'après Creswell et al.).

plexe, caractéristique d'un passage par l'appareil de Golgi [27, 37]. Ce faible niveau d'expression à la surface cellulaire des molécules du CMH-II ne dépend pas de l'haplotype considéré puisqu'un résultat similaire est retrouvé quel que soit l'haplotype étudié $\left(\mathrm{H}-2^{\mathrm{b}}\right.$;

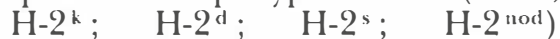
(S. Viville, résultats non publiés).

Dans les endosomes, Ii est clivée par des protéases identiques, au moins pour certaines d'entre elles, à celles responsables de la dégradation de l'antigène, comme par exemple les cathepsines B, D et E [38].

L'inhibition de la protéolyse de Ii au niveau des endosomes empêche le transport des molécules du CMH-II vers la surface cellulaire et l'expression en excès de li bloque les molécules du CMH-II au niveau des endosomes précoces [38, 39].

Toutefois, au cours du transport du complexe $\mathrm{CMH}-\mathrm{II} / \mathrm{Ii}$, certaines molécules de Ii échapperaient à la protéolyse et seraient donc exprimées à la surface cellulaire. Des études faites à l'aide d'un anticorps monoclonal de rat spécifique de Ii mettent $\mathrm{m} / \mathrm{s} n^{\circ} 2$ vol. 10, forrie 94 mes et/ou les lysosomes. Dans les endosomes, les molécules du CMHII libérées de Ii auraient tendance à s'agréger et leur interaction avec un peptide évite l'agrégation [42]. De plus, l'interaction avec un peptide conduit à la formation d'un complexe le plus souvent très stable en présence de détergents, signe d'une interaction forte entre un dimère $\alpha / \beta$ et un peptide dérivé de l'antigène. Là encore, les résultats obtenus avec les souris Ii $^{\circ}$ montrent, qu'en absence de Ii, les molécules du CMH-II exprimées à la surface cellulaire ne présentent pas une stablité maximale [27, 37], probablement parce que leur voie d'exocytose ne croise plus la voie d'endocytose, voie caractérisée par un $\mathrm{pH}$ acide favorable à la fixation des peptides; cependant, il reste à définir si ces molécules du CMH-II sont "vides" ou associées, avec une faible affinité, à un peptide. Enfin, des expériences de transfection de gènes codant pour les molécules du CMHII et Ii suggèrent que, selon l'expression ou non de Ii, les molécules du CMH-II possèdent à la surface cellulaire une conformation différente. Cela a pu être mis en évidence, soit par une différence d'immunoréactivité de certains anticorps monoclonaux anti-molécules du CMH-Il incapables d'interagir avec des molécules du CMH-II synthétisées en absence de Ii [34, 43], soit par une différence dans l'aptitude à stimuler des lymphocytes $T$ alloréactifs, les molécules du C:MH-II exprimées en absence de Ii étant incapables de stimuler de tels clones [43].

Dans ce contexte, il est facile de prévoir le rôle de Ii dans la présentation de l'antigène par les molécules du CMH-II, que ce soit en inhibant la fixation des peptides aux molécules du CMH-II dans le réticulum endoplasmique, ou en contrôlant le transport des molécules du $\mathrm{CMH}-\mathrm{II}$ vers les endosomes ainsi que leur sortie. Stockinger et al. furent les premiers à montrer l'importance des formes p3I et p4I dans la présentation d'antigènes exogènes par les molécules du CMH-II. En effet, alors que la présentation d'antigènes exogènes par des cellules fibroblastiques de souris transfectées par les gènes du C.MH-II nécessite la pré- 


\section{RÉFÉRENCES}

33. Cleasson-Welsh L, Peterson PA. Implication of the invariant $\%$ chain on the intracellular transport of class II histocompatibility antigens. / Immunol 1985; 135: 3551-7.

34. Anderson MS, Miller J. Invariant chain can function as a chaperon protein for class II major histocompatibility complex molecules. Proc Natl Acad Sir USA 1992 89 : 2282-6.

35. Schaiff WT, Hruska KA, McCourt DW, Green M, Schwartz D. HIA-DR associates with specific suress proteins and is retained in the endoplasmic reticulum in invariant chain negative cells. J Fxp Med 1992; 176 657-66.

36. Miller 1, Germain R. Efficient cell surface expression of class Il MHC: molecules in the absence of associated invariant chain. J Exp Med 1986; 164: 1478-89.

37. Bikoff EK, Huang I.Y, Episkopou I', el al. Def ective major histocompatibility complex class Il assembly, transport, peptide acquisition, and $\mathrm{CD}^{+} \mathrm{T}$ cell selection in mice lacking invariant chain expression. I Exp Med 1993; 177 : 1699-712.

38. Neefjes IJ, Ploegh HL. Inhibition of endosomal proteolytic activity by leupeptin blocks surface expression of MHC: class II molecules and their conversion to SDS resistant $\alpha \beta$ heterodimers in endosomes. F.MBO / 1992; 11: 411-6.

39. Romagnoli P, I ayet C, Yewdell J, Bakke O, Germain RN. Relationship between invariant chain expression and major his tocompatibility complex class II transport into early and late endocytic compartment. J Exp Med 1993; 177 : 583-96.

40. Koch N, Moldenhauer G, Hofinann WJ, Müller P. Rapid intracellular pathway gives rise to cell surface expression of the MHC class II- associated invariant chain (C.D74). J Immunol 1991; 147 : 2643-51.

41. Fisch A, Reske K. Cell surface display of rat invariant $\gamma$ chain: detection by monoclonal antibodies directed against a C-terminal $\gamma$ chain segment. Eur / Immunol 1992; 22: 1413-9.

42. Germain RN, Rinker A(;. Peptide bin- invariant-chain free class II dimers and promotes surface expression of occupied molecules. Nature 1993; 363: 725-8.

43. Rath S, Lin RH, Rundensky A, Janeway CA. T and B cell receptors discriminate major histocompatibility complex class Il conformations influenced by the invariant chain. Eur J Immunol 1992; 22 : 2121-7.

44. Stockinger B, Pessara U, I.in RH, et al. A role of la-associated invariant chains in antigen processing and presentation. Cell 1989 ; 56 : $683-9$.

45. Bertolino $\mathrm{P}$, Forquet F, Pont S, et al. Correlation between invariant chain expression level and capability to present antigen to MHC: class ll-restricted T cells. Int Immunol 1991; : 435-43.

46. Nadimi F, Moreno J, Momburg F, et al. Antigen presentation of hen egg-white lvsozyme but not of ribonuclease $A$ is augmented by the major histocompatibility complex class ll-associated invariant chain. Eur J Immunol 1991 ; 21 : 1255-63.

47. Peterson M, Miller J. Invariant chain influences the immunological recognition of MHC, class II molecules. Nature 1990; 345: 172-4.

48. Calin-Laurens V', Forquet F, LombardPlatet S, et al. High efficiency of endogenous antigen presentation by MHC class II molecules. Int Immunol 1992; 4: 1113-21.

49. Lakey EK, Margoliash E, Pierce SK. Identification of a peptide binding protein that plays a role in antigen presentation. Proc Nall Aiad Sci USA 1987; 84 : 1659-63.

50. DeNagel DC; Pierce SK. A case for chaperones in antigen processing. Immunol Today 1992 ; 13 : 86-9.

51. Mellins E, Smith L, Arp B, et al. Def ective processing and presentation of exogenous antigens in mutants with normal HLA class Il genes. Nature 1990; 343: 71-4

52. Mellins E, Kempin S, Smith L., Monji $T$, Pious D. A gene required for class IIrestricted antigen presentation maps to the major histocompatibility complex. / Exp Med 1991; 174: 1607-15. sence de Ii, la présentation des peptides antigéniques est indépendante de Ii [44]. Bien que la présentation d'une majorité des protéines exogènes soit dépendante de Ii [44-46], il existe des exceptions. C'est le cas, par exemple, du peptide OVA 323-339 dérivé de l'ovalbumine d'œuf de poule, présenté à l'hybridome 3DO-54.8 dans le contexte de I-A ${ }^{d}$ [47] ou encore du peptide 43-56, dérivé de la ribonucléase bovine, présenté à l'hybridome TS12 dans le contexte de $I_{-A^{k}}[46]$. Ces résultats ont été confirmés avec les souris $\mathrm{Ii}^{\circ}$ dont les cellules spléniques sont incapables, pour la plupart des antigènes, de présenter des protéines natives, à l'exception de la ribonucléase bovine présentée à l'hybridome TS12 [27]. En outre, la présentation de la ribonucléase à l'hybridome TS12 est inhibée en présence de chloroquine. Ce résultat suggère que, même en absence de Ii, la présentation d'un antigène nécessite un compartiment intracellulaire acide qui intervient, soit dans la formation des complexes molécules du CMH-II/peptide, soit dans la protéolyse de l'antigène.

Dans ce contexte, l'hypothèse suivante peut-être formulée :

- En présence de Ii, la protéolyse de l'antigène et la formation des complexes molécules du CMH-II/peptide ont lieu dans les différents compartiments de l'endocytose: les endosomes précoces, les endosomes tardifs et le compartiment CMH-II. Dans ce contexte, restent à définir les rôles respectifs des formes p31 et p41 ainsi que les mécanismes permettant le transport des complexes molécules du CMH-II/peptides des endosomes tardifs et/ou des compartiments $\mathrm{CMH}-\mathrm{II}$ vers la surface cellulaire.

- En absence de Ii, la protéolyse de l'antigène aurait également lieu dans les différents compartiments de l'endocytose, mais la formation des complexes molécules du CMHII/peptide aurait lieu, quant à elle, dans d'autres compartiments. En effet, dans ces conditions, le nombre de molécules de CMH-II présentes dans les endosomes doit être faible (par absence du motif de ciblage et/ou du motif de rétention inscrit dans Ii). L'interaction entre les pep- 


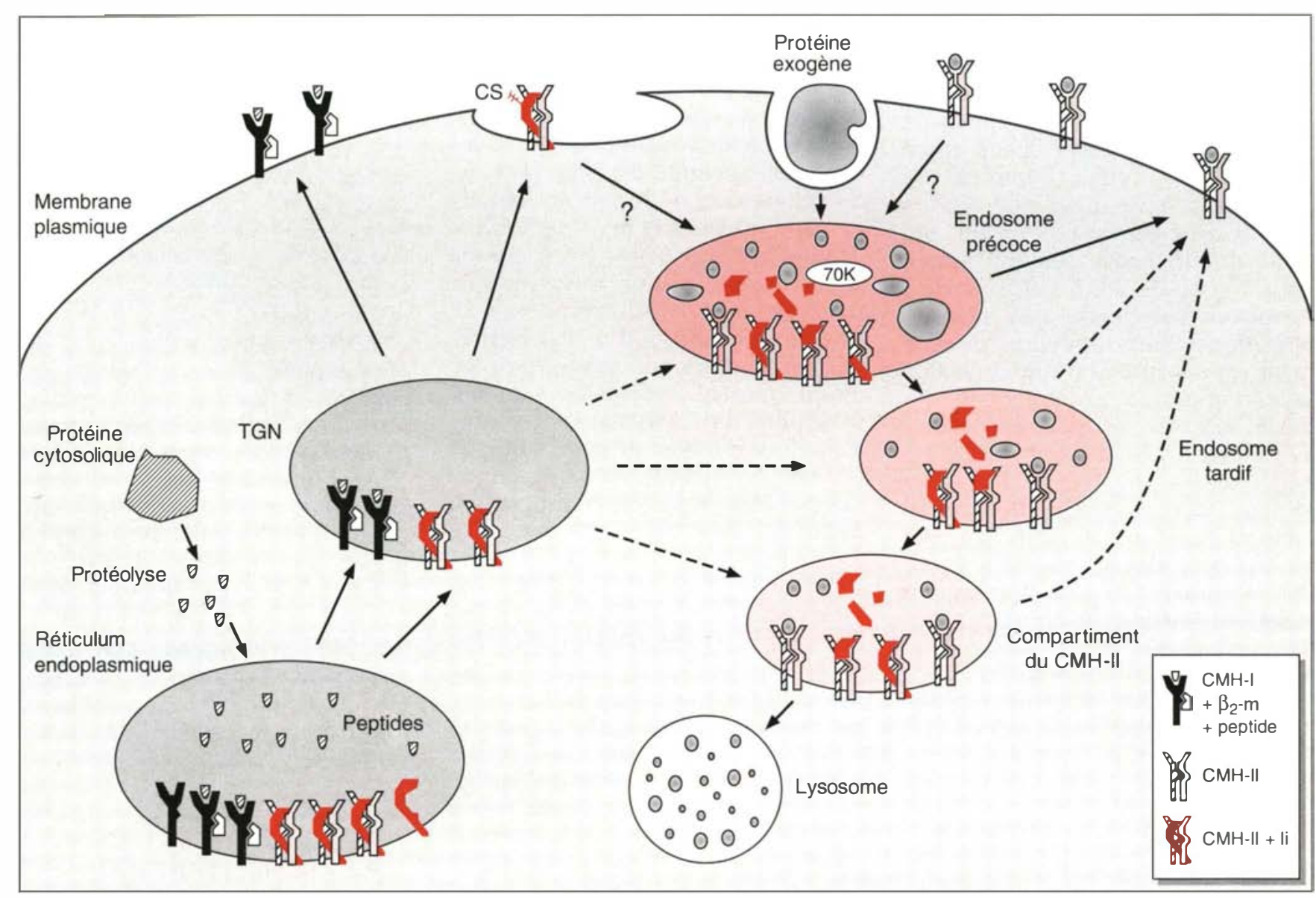

Figure 3. Transport des molécules du CMH à travers la cellule. Dans le réticulum endoplasmique, les molécules de classe I du CMH (CMH-I) forment un complexe tri-partite avec la $\beta 2$-microglobuline $(\beta 2 \mathrm{~m})$ et un peptide provenant de la dégradation protéolytique d'une protéine cytosolique. Ce complexe transite alors par le TGN (trans Golgi network) pour rejoindre la surface cellulaire. Dans le réticulum endoplasmique l'association des molécules de classe II du CMH (CMH-II) avec la chaîne invariante (li) inhibe la fixation de peptides. Une fraction de ce complexe CMH-II/li, celle comportant une li modifiée par un chondroïtine sulfate (CS), vont suivre la même voie de transport que les CMH-I pour parvenir à la surface cellulaire. La majorité des CMH-II, après avoir transité par le TGN, est déviée vers un (des) compartiment(s) de l'endocytose (endosome précoce et/ou endosome tardif et/ou compartiment des $\mathrm{CMH}-1 \mathrm{ll}$. Dans ces compartiments, il est dégradée, libérant les chaînes $\alpha$ et $\beta$ des CMH-II, leur permettant de fixer un peptide provenant de la dégradation d'une protéine antigénique exogène ou d'une protéine endogène membranaire ou sécrétée. C'est ce complexe CMH-II/peptide qui est transporté à la surface cellulaire. Dans les endosomes, une protéine chaperonne 70K interviendrait soit dans l'apprêtement des peptides soit dans leur fixation aux $\mathrm{CMH}-$ II. Les traits en pointillé représentent des voies de transport potentielles.

tides et les molécules du CMH-II pourrait avoir lieu, par exemple, dans le réseau trans- golgien (TGN). Dans ce cas, il faut envisager un transport des peptides des endosomes vers le TGN et/ou une participation des peptides déjà présents dans le TGN. Dans les conditions physiologiques, une interaction au niveau du TGN n'est pas possible car Ii, toujours associée aux molécules du CMH-II dans ce comparti$\mathrm{m} / \mathrm{s} n^{\circ} 2$ vol. 10 , férier 94 ment, inhibe la fixation des peptides (figure 3).

La chaîne li joue également un rôle important dans la présentation des antigènes endogènes, c'est-à-dire synthétisés par la cellule présentatrice de l'antigène. En effet, la présentation, par les molécules du CMH-II, d'antigènes endogènes membranaires et secrétés est particulièrement efficace [48] et la présentation du peptide HEL 46-61 dérivé du lysozyme de poule par les molécules $\mathrm{CMH}-\mathrm{II}$ I- $\mathrm{A}^{\mathrm{k}}$ est dépendante de Ii, que l'antigène HEL soit apporté à la cellule présentatrice de l'antigène sous forme exogène ou sous forme endogène [14]. L'utilisation des souris $\mathrm{Ii}^{\circ}$ a également montré que la présentation d'un peptide dérivé de IgG2a nécessite Ii, que l'IgG2a soit apportée à la cellule présentatrice de l'antigène sous forme exogène ou sous forme endogène [37]. 


\section{Conséquences} de l'absence de li in vivo

La création de souris déficientes en chaine Ii a permis d'étudier son rôle non seulement in vitro mais aussi in vivo. Pour ce faire, deux approches ont été utilisées. La première approche a été d'étudier l'aptitude des souris $\mathrm{Ii}^{\circ}$ à développer une réponse humorale spécifique pour un antigène donné. Curieusement, il a été montré que de telles souris ne développaient pas de réponse primaire spécifique alors qu'elles développent, après une seconde injection, une réponse secondaire quasi normale [27]. La seconde approche a été d'étudier la sélection positive et négative des lymphocytes T. Les études de cytofluorimétrie de flux mettent en évidence un déficit de sélection positive des lymphocytes $\mathrm{T}$ auxiliaires caractérisés par le marqueur CD4. En effet, aussi bien au niveau du thymus que de la périphérie, il est constaté une diminution importante du nombre de lymphocytes $\mathrm{T}$ $\mathrm{CD}^{+}[27,37]$. La population $\mathrm{CD}^{+}$ se retrouve, quant à elle, augmentée dans une proportion similaire [27]. Cette diminution dans la sélection positive des cellules $\mathrm{CD}^{+}$pouvant être due, soit à la faible proportion des molécules du CMH-II exprimées à la surface cellulaire, soit à la conformation aberrante de ces molécules du CMH-II, soit à la combinaison des deux. La sélection négative semble, elle aussi, affectée par cette mutation dans la mesure où il a été constaté une diminution de la délétion des lymphocytes $\mathrm{T}$ exprimant des TcR particuliers, notamment ceux dont la chaîne $\beta$ est capable d'interagir avec un superantigène [27]. Cela suggère encore une fois que la conformation des molécules CMH-II est modifiée en absence de Ii.

\section{Perspectives}

Dix ans après sa découverte et bien que de nombreuses questions posées restent sans réponse, l'importance de la chaîne Ii dans la réponse immunitaire spécifique est clairement démontrée. Il reste à définir le rôle respectif des différentes formes de Ii et des modifications post- traductionnelles dans la présentation antigénique et/ou le transport des molécules du CMH-II ainsi que l'importance et le rôle de l'expression de surface de Ii.

La dysrégulation de l'expression de la chaîne Ii pourrait avoir des conséquences sur l'apparition de maladies auto-immunes. En effet, la perte de l'expression de Ii pourrait entraîner une stimulation des lymphocytes T autoréactifs présents, en réponse, soit à la conformation aberrante des molécules du CMH-II exprimées à la surface cellulaire, soit à la présentation de peptides qui ne sont pas, en temps normal, présentés par les molécules du CMH-II.

Toutefois, d'autres protéines que la chaîne Ii jouent un rôle dans l'assemblage et dans le transport des molécules du CMH-II. Par exemple, la protéine chaperon PBP72/74, membre de la famille des heat shock proteins [49], faciliterait l'interaction des molécules du CMH-II avec les peptides [50]. Enfin, un phénotype ressemblant à celui des souris $\mathrm{Ii}^{\circ}$ a été décrit pour une lignée cellulaire (16.23) exprimant Ii [51] : cette lignée cellulaire est incapable de présenter des antigènes natifs et les molécules du CMH-II à la surface cellulaire ne possèdent pas une conformation stable [52]

\section{Remerciements}

S. Viville souhaite remercier la Fondation Marcel Mérieux ainsi que l'Association pour la Recherche contre le Cancer pour leur aide financière.

\section{TIRÉS A PART}

S. Viville.

\section{Summary}

Invariant chain: role and function in the immune response

Invariant chain is a monomorphic protein, closely associated to the major histocompatibility complex (MHC) class II molecules, that exists in multiple forms encoded by a single gene. Ten years after its discovery, the role of the invariant chain in immune response has been established. In vitro experiments have shown its role in the assembly of the MHC. class II $\alpha$ and $\beta$ chains and in their transport from the endoplasmic reticulum to the endosomes. In addition, the involvement of the invariant chain in antigen presentation by MHC class II has been demonstrated for the majority of antigens. Recently, the creation of invariant chain deficient mice confirmed in vitro studies and now allows for further investigation of its in vivo function. 